\title{
EDUCAR EN CONTEXTO \\ DE SEGREGACIÓN TERRITORIAL. \\ EL CASO DE BAJOS DE MENA
}

\section{Educate in the context of territorial segregation. The case of Bajos de Mena}

\author{
Daniela Maturana Castillo \\ Universidad de Santiago de Chile \\ Correo-e: daniela.maturana@usach.cl \\ Paulina Fernández SÁnchez \\ Colegio Notre Dame. Santiago de Chile \\ Correo-e:pfernandez@notredame.cl \\ Recibido: Io de diciembre de 2019 \\ Envío a informantes: 20 de diciembre de 2019 \\ Aceptación definitiva: 25 de abril de 2020
}

Resumen: Explicar el sistema educacional chileno y la educación en contextos de exclusión y desigualdad requiere de un análisis que supere las condiciones educativas y se adentre en un plano de mayor profundidad sobre la segregación. En este artículo se explora la relación entre políticas habitacionales y educacionales implementadas de la mano del sistema neoliberal a partir de la década de 1980 hasta la actualidad en Chile, particularmente en la localidad de Bajos de Mena, Santiago. Considerando estas políticas y su relación en la definición de la desigualdad, se analiza la complejidad que supone educar en estos contextos, a partir del análisis de contenido aplicado al relato de un profesor de una escuela del sector, donde la relación laboral y educativa se condiciona por la violencia y la distancia simbólica entre estudiantes y profesores.

Palabras Clave: desigualdad; segregación; exclusión; marginalidad.

Aвstract: Explaining the Chilean educational system and education in contexts of exclusion and inequality requires an analysis that goes beyond educational conditions and goes deeper into segregation. This article explores the relationship between housing and educational policies implemented by the neoliberal system from the 1980 os to the 
present in Chile, particularly in the town of Bajos de Mena, Santiago. Considering these policies and their relationship in the definition of inequality, the complexity of educating in these contexts is analyzed, based on the analysis of content from the story of a school teacher in this sector, where the labor and educational relationship is conditioned for the violence and the symbolic distance between students and teachers.

KEY WORDS: inequality; segregation; exclusion; marginality.

\section{Introducción}

$\mathrm{L}$

A DESIGUALDAD ES UN FENÓMENO COMPLEjo en la medida que se produce y reproduce en torno a un imbricado sistema de variables que se intervienen entre . El caso particular de Chile, las desigualdades son evidentes y ampliamente documentadas (Contreras, 1999). Estudios recientes (PNUD, 2017) han avanzado hacia la definición de factores que explican este fenómeno, considerando ámbitos tan relevantes como el ingreso, el trato, el acceso a la cultura, la vivienda, el acceso al poder y la historia familiar, ligada a linajes que han consolidado una oligarquía tradicional que remonta sus orígenes a la conquista de América.

Sin embargo, al fotografiar una determinada realidad, pareciera que ciertas variables adquieren un peso específico mayor. En este caso, comprender los bemoles que implica educar en contextos de exclusión social, dos variables emergen como ámbitos fundamentales para el análisis. La primera de ellas se representa en torno a la expresión territorial, producida y reproducida a través de políticas habitacionales que dibujan claras fronteras y normas de apropiación de los espacios. La segunda variable se erige en torno a los mecanismos de apropiación, transmisión y reproducción cultural generados a partir de las políticas educacionales que dan vida a un sistema educativo con claras particularidades territoriales.

La conjugación de estas dos variables permite entender el fenómeno que se da en el sector de Bajos de Mena en Santiago de Chile. Comprender este caso requiere un análisis de las políticas habitacionales y educacionales implementadas en los últimos cuarenta años, a partir de la imposición de un sistema económico neoliberal en contexto de una democracia tutelada. Antes de profundizar en este caso, merece la atención explicar cómo las dos variables enunciadas se han desarrollado en Chile desde los años ochenta y consolidado a partir del advenimiento de la democracia hasta la actualidad.

\section{La segregación social y territorial en la ciudad de Santiago de Chile}

La escasez de la vivienda fue un ámbito de preocupación y tensión sobre el espacio urbano de la ciudad de Santiago y sus alrededores, entre la segunda mitad del siglo XIX y la primera del siglo xx, con los procesos de migración campo ciudad desarrollados durante prácticamente un siglo. Este proceso generó gran presión por terrenos habitables, lo que devino en la ocupación ilegal de terrenos en diversos puntos de la ciudad, propiciando condiciones de habitabilidad precarias e insalubres, lo que constituyó una de las principales características de lo que se ha denominado la cuestión social (Grez, I995). 
Por esto, el acceso a la vivienda ha sido un tema de preocupación a lo largo de la historia de Chile, tanto para los habitantes desplazados y allegados a espacios de difícil habitabilidad, como para el Estado, responsable de garantizar condiciones de vida mínimas desde la perspectiva de la dignidad humana.

Durante el siglo xx se desarrollaron e impulsaron diversas políticas orientadas a dar respuestas a las necesidades habitacionales, en especial considerando las demandas de la nueva clase trabajadora (otrora población rural) y el emplazamiento al Estado que significaron las poblaciones que se empezaron a consolidar en torno a asentamientos informales, de autoconstrucción y altamente precarizados, como los llamados campamentos. Esta dinámica marca el origen de una marginalidad expresada en el territorio e intencionada por el Estado, lo que propugna que los sectores de menos recursos se concentren y aíslen de los centros urbanos, sus servicios y los espacios de toma de decisión, generando condiciones de vida diferenciadas entre el centro y la periferia.

Así, desde la Ley de Habitaciones Obreras (1906), la Ley de Habitaciones Baratas (1925), la Caja de Habitación Popular (1936) y las políticas desarrolladas entre los años 60 y 70 , se buscó dar respuestas a la demanda habitacional de colectivos de trabajadores y, posteriormente, a colectivos organizados de pobladores, ocupando grandes extensiones de terrenos baldíos disponibles en las áreas más alejadas de la ciudad para generar estas soluciones.

Particularmente durante ese último periodo (I970-I973), en clara coherencia con una política de desarrollo social, se intentó avanzar hacia el desarrollo de conjuntos habitacionales más armónicos, que desincentivaran la autoconstrucción con el fin de asegurar condiciones de calidad de vida homogéneas para sus habitantes. Inclusive, durante esos años se generaron importantes facilidades para que los sectores medios accedieran a viviendas de muy buena calidad.

Pese a estos intentos por facilitar el acceso a la vivienda, el advenimiento del neoliberalismo impactó en la ocupación del espacio y en las políticas habitacionales implementadas a partir de la década de los ochenta, y con mayor fuerza a partir de la década siguiente. Este cambio ideológico promovió la privatización y la acción regulatoria del mercado para todos los ámbitos de la vida. El rol subsidiario del Estado adquiere una evidente expresión territorial, lo que se traduce en cambios en las políticas habitacionales implementadas hasta ese periodo (Tapia, 20II). Esta reorientación del Estado adquiere dos formas gravitantes para analizar las políticas habitacionales: la concepción del espacio y la visión de los sujetos carentes de vivienda como individuos y no como colectivos.

En la primera forma, las políticas coordinadas por el Estado a través del Ministerio de Vivienda y Urbanismo impulsaron a partir de la década de los ochenta y a través de empresas privadas la construcción de soluciones habitacionales de alto volumen de unidades, pero de bajo costo y pocos metros cuadrados (el tamaño fluctuaba entre 40 y 45 metros), con bajos estándares de calidad de las viviendas, emplazadas en los lugares periféricos de la ciudad ${ }^{\mathrm{I}}$, carentes de todo tipo de equipamiento urbano y con un limitado (o a veces inexistente) número de servicios básicos, generando con ello una condición de segregación territorial y funcional del espacio

En este escrito, las políticas habitacionales están centradas en la realidad de la ciudad de Santiago particularmente. 
urbano (Hidalgo, 2007). Esto permitió que las empresas constructoras aumentaran sus ganancias en detrimento de la calidad de vida de sus habitantes.

A pesar de todos los reparos que recibe esta política de construcción, esta efectivamente logró disminuir el déficit de viviendas que se arrastraba de décadas anteriores, por lo que, en cierto punto, se calificó como una respuesta eficiente frente al problema (Hidalgo, 2007).

Sin embargo, estas medidas significaron reacomodo y desplazamientos de grandes masas de población, que llegaron a habitar estos nuevos espacios ${ }^{2}$. De hecho, al analizar la localización de la construcción de las viviendas sociales a partir de los años 80 en adelante, las cifras son elocuentes. Para más precisión, cabe señalar que, entre 1990 y 2002, el 49,48\% de las viviendas sociales construidas se ubicaron en la comuna de Puente Alto, y solo in comunas de las 33 que componen el Gran Santiago recibieron a estos nuevos habitantes: los erradicados (Hidalgo, 2007).

Por otra parte, estas políticas habitacionales se caracterizaron por tratar a las personas que demandaban la tenencia de un hogar como sujetos y no como colectivos. Las políticas de subsidios habitacionales premiaron el ahorro individual y transformaron a estas personas en consumidores, contribuyendo a la desarticulación de los comités por la vivienda (Tapia, 20II). De esta forma, los erradicados -como se denominó a las familias que eran trasladadas desde los sitios de ocupación ilegal a sus nuevas viviendas- se vieron en muchos casos desprovistos de sus redes sociales, desplazados a nuevos sitios, muy lejanos del centro de la ciudad, con escaso acceso al transporte y los servicios.

Este panorama prontamente se transformó en desolador, y muchos de estos sectores se vieron invadidos por la criminalidad y la criminalización desde los sectores sociales más acomodados, transformando en un estigma el provenir desde una población.

Por lo tanto, la erradicación de campamentos y el desplazamiento a barrios periféricos lejanos de todo servicio, en conjunto con la ruptura del tejido social, hicieron de estas nuevas poblaciones espacios para el desarrollo de formas de habitabilidad y procesos socializadores propios, particularmente para la población juvenil de esos espacios (Ortega, 2014). Es decir, el gueto se transforma en una unidad en sí misma, aislada del resto de la ciudad y, por lo tanto, comienza a desarrollar patrones de conducta impropios a los ojos del resto de la población que está fuera del gueto, pero dentro de la ciudad. En términos de Bourdieu (1997), los habitantes de estos lugares no comparten habitus de los habitantes con más ingreso, lo que tiene una inminente repercusión espacial.

A su vez, y como una plausible consecuencia de este habitus no compartido, la estigmatización y criminalización de los jóvenes de las poblaciones se asocia a una de las condiciones de desigualdad de trato reproducidas en y por la sociedad chilena (PNUD, 20I7). De esta misma forma, la legislación de los últimos años ha ido en el sentido de la persecución de los jóvenes que cometen actos delictuales. Esto, a partir de la implementación de un sistema de responsabilidad penal desde los I4 años (ley promulgada en el año 2005) y, posteriormente, la ley de violencia escolar, implementada principalmente

Incluso, para quienes no se vieron «beneficiados» y por lo tanto no se desplazaron, recibieron en sus viviendas las denominadas casetas sanitarias, entendidas como construcciones que solo aseguran el acceso a la red de alcantarillado ( 9 a I2 metros cuadrados donde se localiza un baño y cocina) y por lo tanto vuelven a estimular la autoconstrucción. 
en los colegios municipales. En ambos casos, estas leyes han contribuido al desarrollo de un escenario sociopolítico de criminalización de los jóvenes como sujetos, ignorando los condicionantes sociales, educacionales y territoriales que influyen en el desarrollo de conductas más violentas o de infracción de ley. Mas bien, se orientan a la «detección temprana» de futuros delincuentes (Morales y López, 20i6).

En definitiva, el espacio urbano de Santiago a lo largo del siglo xx, y con énfasis a partir del advenimiento del modelo neoliberal, es la expresión de la marginalidad como política de desarrollo urbano y organización territorial.

\section{Sistema educacional en Chile: privatización y segregación}

El sistema educacional en Chile presenta evidentes características de desigualdad (PNUD, 20I7). Durante la dictadura militar se produjeron grandes cambios estructurales que con el correr del tiempo y la llegada de la democracia no fueron modificados, sino que se profundizaron y consolidaron.

A principios de los años 7o, durante el gobierno de Salvador Allende, se construyó el proyecto de la Escuela Nacional Unificada (ENU), que básicamente buscaba la transformación de la educación instalando como consigna que esta debía ser permanente, democrática, participativa, pluralista y acorde con las necesidades económicas del país y, evidentemente, de responsabilidad del Estado (Ministerio de Educación Pública, 1973). Esa idea, que no llegó a materializarse, se interrumpe, paraliza y luego se modifica estructuralmente con la imposición del modelo neoliberal y, posteriormente, con la promulgación de la Constitución Política de i980.

Por lo anterior, el sistema educacional en Chile es resultado de una fuerte segregación y participación de agentes privados en los procesos de educativos. Las políticas tendientes a la privatización de la educación establecen la organización de un sistema de educación escolar en tres tipos de escuela que, a su vez, obedecen a tres tipos de dependencias: aquellas dependientes de los municipios, las llamadas escuelas municipales; las que se financian a través de capital privado y por medio de un voucher de parte del Estado, denominadas particulares subvencionadas, y, por último, aquellas que en su totalidad se financian con capital privado, las llamadas escuelas privadas, las cuales equivalen a menos del Io\% de los establecimientos escolares de Chile (Gallego y Sapelli, 2007; Ministerio de Educación, 2018).

Esta distribución segregada contribuye a fortalecer un sistema educacional condicionado a la capacidad de pago de las familias y a la ubicación de estas escuelas. Esto se puede afirmar porque los agentes intervinientes se regulan en función del comportamiento del mercado, dado que i) el financiamiento es a la demanda y 2) los dueños de los establecimientos son privados, por lo tanto, la decisión sobre dónde construirlos opera con escasa regulación estatal. Así, la escuela se decide en función de los intereses de los privados que operan como dueños de estos espacios, alejados de la lógica gubernamental (Bellei, González y Valenzuela, 2oıо).

Por lo tanto, en términos generales, las escuelas de mayor calidad se instalaron donde existía una mayor población con capacidad de pago, dando cuenta de la desigualdad en el acceso a la educación como un ámbito asociado a la segregación socioeconómica en Chile. Hay que tener en consideración que este sistema se basa en el principio básico -asegurado constitucionalmente- de que son los padres los 
encargados de decidir la educación de sus hijos y, por lo tanto, de elegir la mejor escuela para ellos (Cornejo et al., 20I5). Sin embargo, las evidentes desigualdades sociales, educacionales y económicas de los padres inciden en que estos no estén en planos equivalentes a la hora de decidir la educación de sus hijos y, por lo tanto, tiendan a acceder a la escuela más próxima. Es decir, emerge nuevamente la variable territorial.

El voucher -prácticamente ilimitado, suplementable y no restringido- instalado en Chile (Gallegos y Sapelli, 2007) actuó como un claro factor coadyuvante en la desigualdad (Sabatini y Wormald, 2013), ya que, mientras más capacidad de pago en las familias, más distancia tomaron de las escuelas públicas, disminuyendo progresivamente la matrícula de estas a favor de las escuelas con financiamiento compartido.

Por otra parte, considerando que las escuelas públicas en realidad son de responsabilidad de los municipios, el acceso a los recursos en estas escuelas también difiere. A partir del análisis de Bravo (20I4), se establece que las fuentes de financiamiento municipal dependen fuertemente de impuestos y cobros en base al valor patrimonial de los activos en la comuna, propiciando procesos de reproducción de la riqueza/pobreza.

De allí que, frente a la decadencia y la necesidad de fomentar este tipo de dependencia escolar, recién a partir del 2007 y hasta la actualidad, comienzan a generarse cambios a nivel de políticas públicas orientadas a revertir en parte la desigualdad del modelo, aunque sin modificar su estructura basada en la competencia entre privados.

Ahora bien, cabe señalar que esta era de reformas desarrollada en la última década es producto de la irrupción del movimiento social protagonizado por los estudiantes secundarios en el año 2006 -en la llamada revolución pingüina- y en la consecuente movilización universitaria del año 20II (Garcés, 20I2).

Dentro de las medidas adoptadas en este proceso reformista (Pardo, 2013), se apostó por acciones orientadas a avanzar en materia de aumento de recursos, diferenciando el financiamiento en función del nivel socioeconómico del estudiantado que se atiende; supervisar la calidad educativa, a partir de la creación de nueva institucionalidad; conceder gratuidad para el sistema de educación terciaria en función de quintiles de ingreso de los estudiantes; restringir la selección en establecimientos que reciben recursos del Estado; fortalecer la educación pública a partir de la definición de servicios locales de educación, los que de manera progresiva (hasta el 2030) reemplazarán a los establecimientos municipales; fortalecer y normar la carrera docente, para que la formación de profesores responda a las demandas de calidad provenientes de la eclosión social.

Específicamente en torno al aumento de recursos para financiar la educación municipal y particular subvencionada, a partir del año 2008 se crearon medidas que apuntaron a la mejora del sistema de subvención. Se introdujo la Subvención Escolar Preferencial (SEP), que tuvo como propósito otorgar mayor equidad en educación, aumentando el aporte estatal cuando las escuelas aumentaban la matrícula de estudiantes prioritarios por su alto nivel de vulnerabilidad. Adicionalmente, se creó el fondo de apoyo al mejoramiento de la gestión municipal en educación, con el objeto de corregir en parte las desigualdades entre municipios de mayores y menores recursos (EDUCACIÓN 2020, 2017). Sin embargo, ya desde sus primeros momentos de implementación, se esgrimió que la implementación de la Ley SEP no aseguraba mejoras en las escuelas más vulnerables, ya que esta ley supone un grado de competencia 
técnica para su implementación que no siempre está disponible en los territorios más desposeídos (Weinstein, Fuenzalida y Muñoz, 2oro).

Más tarde, en el año 20I5, se promulgó la Ley de Inclusión Escolar (n. ${ }^{\circ} 20.845$ ), que apuntó a transformar el sistema de educación chileno bajo tres pilares: fin al lucro; fin al copago, lo que pretende eliminar paulatinamente el aporte que realizan las familias a los establecimientos, en la medida que el Estado entrega más fondos; y regulación de la admisión escolar, a través de un sistema único y centralizado de admisión que elimina los sistemas injustos de admisión, donde uno de los más importantes criterios de ingreso son los resultados en pruebas de admisión.

A pesar de esto, para el año 2018 la matrícula en establecimientos de dependencia particular subvencionada continuaba siendo mayoritaria en el sistema educativo chileno, concentrando el $53,8 \%$ del total nacional. Le siguen las dependencias municipal y particular pagada, que cuentan con el segundo y tercer lugar de concentración de matrícula, con una distribución del $34,3 \%$ y un 9,1\% respectivamente, según cifras del Ministerio de Educación (MINEduc, 20I8).

Por otra parte, esta segregación asociada a la capacidad de pago tiene una clara repercusión en la distribución de las escuelas que logran mejores resultados en las evaluaciones estandarizadas aplicadas a nivel nacional ${ }^{3}$.

Teniendo en cuenta lo anterior, el Sistema de Medición de la Calidad en la Educación (SIMCE) 4 y la Prueba de Selección Universitaria (PSU) actúan como muestra plausible de la efectividad del proceso de enseñanza aprendizaje durante la formación escolar, evidenciando el peso de las variables socioeconómicas y territoriales. A mayor capacidad de pago y asentamiento en las comunas con más ingreso, mejores resultados obtenidos. Los datos oficiales son categóricos: la variable socioeconómica sigue siendo un factor diferenciador respecto a los niveles alcanzados en esta prueba.

Claro ejemplo de ello es lo que revela el análisis de los puntajes sImce. Tanto en el SIMCE de Lectura como en el de Matemática, a medida que se aumenta el nivel socioeconómico se presenta una mayor proporción de estudiantes que alcanzan el nivel Adecuado, mientras que, en los niveles socioeconómico más bajos, un mayor porcentaje de estudiantes se localiza en el estándar Insuficiente. Particularmente en el caso de la prueba de Lectura aplicada en $4 .^{\circ}$ básico, un $47 \%$ del estudiantado de clase baja se encuentra en el nivel Insuficiente, porcentaje que aumenta a un $63 \%$ en la misma prueba en $8 .^{\circ}$ básico. En cambio, solo un $10 \%$ de los alumnos y alumnas de clase alta que cursan $4 .^{\circ}$ básico se ubica en este estándar, mientras que un $68 \%$ de estos alumnos y alumnas está en el estándar Adecuado. Estas diferencias son aún más evidentes en el SIMCE de Matemática, donde el 58\% de los estudiantes de clase baja de $4 .^{\circ}$ básico mostraron un nivel Insuficiente, proporción que disminuye a un $8 \%$ entre las clases altas (MINEDUC, 20I8).

Para el caso de este artículo, se tomarán como referencia de la calidad educativa los puntajes obtenidos en pruebas estandarizadas, aunque se reconoce que esta no solo depende de aquellos resultados, pero que de todas formas entrega evidencia sobre el grado de cobertura curricular que alcanzan las escuelas.

4 El Sistema de Medición de la Calidad de la Educación (SIMCE) fue creado en 1988 con el objetivo de institucionalizar una evaluación estandarizada para todo el sistema educacional chileno. Evalúa el logro de los contenidos y habilidades del currículo vigente, en diferentes asignaturas o áreas (consideradas por el sistema como más relevantes y a las que se destina mayor número de horas), a través de una medición que se aplica a todos los estudiantes del país que cursan los siguientes niveles: cuarto, sexto y octavo año básico y segundo medio. 
Por lo tanto, a la luz de estos datos, es posible señalar que la mayor inyección de recursos suscitada a partir de las reformas de la última década no ha logrado orientar un sistema de educación igualitario y equitativo, más bien ha hecho que el municipio donde se emplazan las escuelas y el nivel socioeconómico de sus vecinos sigan siendo muy determinantes a la hora de acceder a recursos, tener demanda de matrículas y obtener resultados de calidad, lo que, a su vez, se relaciona directamente con las iniciativas de las autoridades locales y, por ende, de cada municipio, lo cual replica la desigualdad territorial existente en el país.

Esta organización del sistema escolar, pese a todo el proceso reformista iniciado hace una década y aún en franco desarrollo, se orienta a lograr una mayor equidad en el acceso a la educación y calidad educativa. Sin embargo, el consenso social es que, a pesar de estos esfuerzos, la calidad sigue estando atribuida a la capacidad de pago de las familias, fuertemente correspondidas con la expresión territorial asociada a la distribución de la riqueza.

En definitiva, se genera una relación directamente proporcional entre políticas habitacionales de los años 90 que establece que en comunas acomodadas los habitantes acceden a mejor educación, mientras que las comunas ubicadas en sectores periféricos y vulnerables poseen ofertas de escuelas de baja calidad, asociadas al pago, lo que evidencia una relación indisociable entre escuela y territorio.

\section{Bajos de Mena como un gueto urbano}

Bajos de Mena es la expresión territorial de un ejemplo claro de precarización y marginalidad producto de las políticas habitacionales implementadas desde los años 80 y 90 en Chile. Es un asentamiento ubicado en los márgenes de la ciudad de Santiago, específicamente en la comuna de Puente Alto, a 20 kilómetros del centro de la ciudad. A principios de los años 8o, este lugar era un terreno baldío de propiedad del arzobispado. Su distancia respecto del centro de la ciudad y su ubicación «incógnita» para las clases acomodadas lo transformaron en un sitio propicio para la formación de un vertedero ilegal.

Sin embargo, con el paso del tiempo, su extensión fue valorada como favorable a la política habitacional de la época, ya que posibilitaba la construcción de un gran número de viviendas de bajo costo. Por esta razón, ya al iniciar la década de los noventa, el Estado de Chile compró el terreno y comenzó la construcción de viviendas sociales, orientadas a la reubicación de familias.

Como muestra de las políticas en materia de vivienda, el Estado adquiere el terreno con el fin de otorgar solución habitacional para familias vulnerables, pero no construye directamente. Esta labor se externaliza a través de empresas constructoras, que, de manera independiente y sin ningún plano regulador de por medio, crean villas desarticuladas entre sí, inconexas y carentes de servicios. Es decir, promueve el mercado de la vivienda social desregulada y no planificada (Puchi, 20I7).

La precariedad que este escenario supone se potencia en el caso de Bajos de Mena, debido a que sus límites son claros e inescrutables. La construcción de modernas autopistas urbanas en sus alrededores -como muros infranqueables- contribuye al distanciamiento, la inconexión y el aislamiento de este espacio respecto del resto de la ciudad (Puchi, 20I7; Martínez, 2017). 
Por otra parte, la calidad de la vivienda en Bajos de Mena es una clara expresión de la desigualdad de la ciudad de Santiago, transformándose en un paradigma de segregación y marginación urbana (Hidalgo, Urbina, Alvarado y Paulsen, 20I7) y de viviendas de mala calidad. Por ello, emerge desde sus habitantes la representación simbólica de que sus hogares son similares a una caja de cartón (Martínez, 2017).

Por lo tanto, Bajos de Mena configura un territorio donde realmente la lógica segregacionista ha funcionado, convirtiéndose en un gran gueto urbano, que opera y reproduce lógicas que en nada comulgan con formas de interacción. Constituye un espacio materialmente segregado y excluido de la ciudad y, al mismo tiempo, representa en el ideario urbano un espacio de criminalidad que estigmatiza a sus habitantes. De esta forma esta segregación, en su condición material y simbólica, relega a sus habitantes a un espacio carenciado y vulnerado, abandonándolos a su suerte.

La figura muestra la distancia de este asentamiento respecto del centro de la ciudad.

Figura i. Ubicación de Bajos de Mena en el Gran Santiago

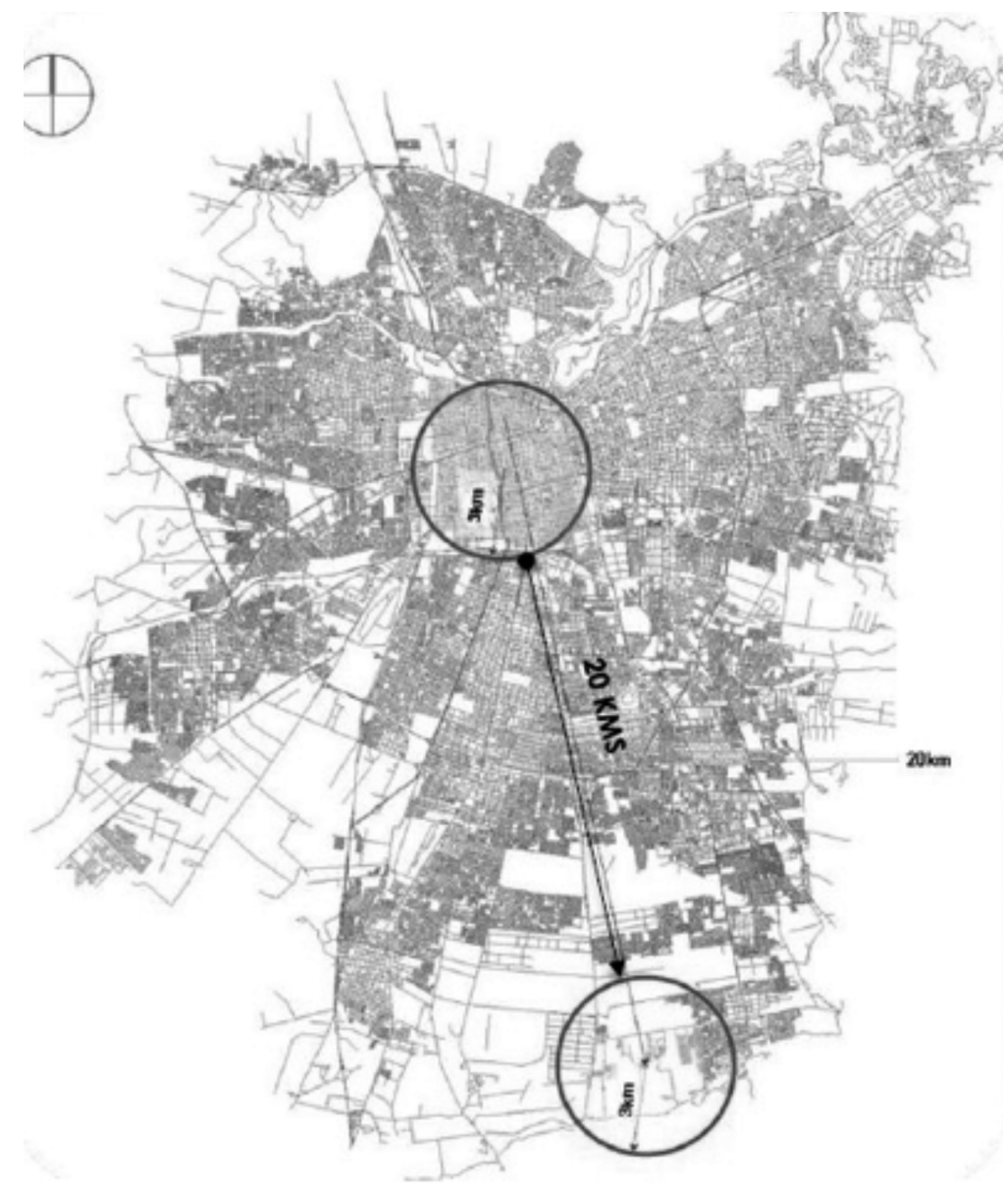

Fuente: PuCHI, 2017. 
Por las razones esgrimidas, comprender la complejidad de las escuelas en este contexto requiere revisar la articulación de las políticas habitacionales y educacionales, las que en conjunto dan vida a una realidad particular.

Como se ha abordado, el sistema educacional en Chile no es ajeno a la segregación territorial promovida de la mano de la inserción del modelo neoliberal. La completa libertad de los mercados ha provocado tras décadas del desarrollo neoliberal que tanto el suelo como la educación se aprecien y valoricen como bienes de consumo, reproduciendo una ciudad profundamente desigual.

\section{La vivencia de educar el contexto de segregación. Un caso en Bajos de Mena, Puente Alto}

Entender procesos y vivencias que se desarrollan en contextos de exclusión social implica analizar las diversas variables que en ellos repercuten. Bajos de Mena es un caso paradigmático de la exclusión. Por décadas sus habitantes, principalmente erradicados de otras zonas de Santiago, han vivido en completo abandono. Ese abandono sin duda implica la falta de servicios o la mala calidad de ellos.

Respecto a los servicios educativos emplazados en el territorio de Bajos de Mena, alrededor del ı2\% corresponden a escuelas municipales's. Si bien la totalidad de estas escuelas son gratuitas (copago o), alrededor del $88 \%$ corresponden a escuelas privadas que se financian a través de los aportes del Estado y de los recursos adicionales brindados bajo el marco de la Ley sEP.

Por lo tanto, a pesar del no pago y actualmente la No selección académica, es claro que el Estado no ha intervenido directamente en la conducción de comunidades escolares, abandonando la Educación Pública (Atria, 2010) y dejando nuevamente que agentes privados eduquen a una población altamente segregada, marginada y criminalizada. Es más, el rol del Estado se ha limitado a medir y comparar estas escuelas en función de los indicadores (principalmente resultados SIMCE) con los que se mide la efectividad del proceso educativo en cualquier otro sector del país.

Sin embargo, resulta interesante indagar sobre las valoraciones y percepciones que emergen desde estos territorios escolares. Esto permite una aproximación sobre lo que implica educar en estos contextos. Para ello, se recupera la voz de uno de estos profesores, inserto en una de las escuelas particulares subvencionadas emplazadas en Bajos de Mena, propiedad de una corporación cristiana.

El análisis de esta voz no pretende en nada generalizar ni explicar la complejidad del proceso educativo en una zona como la descrita. Los alcances de esta intervención son mucho más acotados y discretos. Simplemente, el propósito radica en rescatar la voz de un protagonista que, desde la primera línea, construye una descripción de lo que implica la docencia, avanzando hacia una territorialización del fenómeno educativo. Nuevamente, el territorio tensiona al sistema educativo y lo emplaza para una respuesta inequívoca.

Para rescatar esta voz se recurrió a la siguiente metodología. El profesor fue entrevistado en profundidad al término del año 2017. La entrevista fue transcrita de manera

\footnotetext{
Dato calculado en función de la información disponible sobre las escuelas de la comuna de Puente Alto en http://www.mime.mineduc.cl/mime-web/mvc/mime/listado. Fecha de consulta: or de diciembre, 2019 .
} 
literal y sometida a análisis de contenido en función de ciertas categorías teóricas en un contexto de análisis intensivo (Piñuel, 2002), dibujando códigos analíticos que permitieran una mejor comprensión del universo simbólico del docente (Gibbs, 20I2). Si bien este ha sido el marco general, se ha dado espacio para recabar categorías emergentes (Strauss y Corbin, 2002) ya que ello permite posicionar y comprender de una manera más holística el universo del actor y su discurso.

De esta forma, estas categorías emergen de la literatura, articulando marcos conceptuales propios de la sociología educacional y de la geografía, ya que, como se ha sostenido, comprender la complejidad de una escuela en Bajos de Mena requiere de la conjunción de estos universos conceptuales.

Estas categorías se agrupan en la siguiente red conceptual:

Figura 2. Elaboración propia con uso de Software Atlas ti 7.0

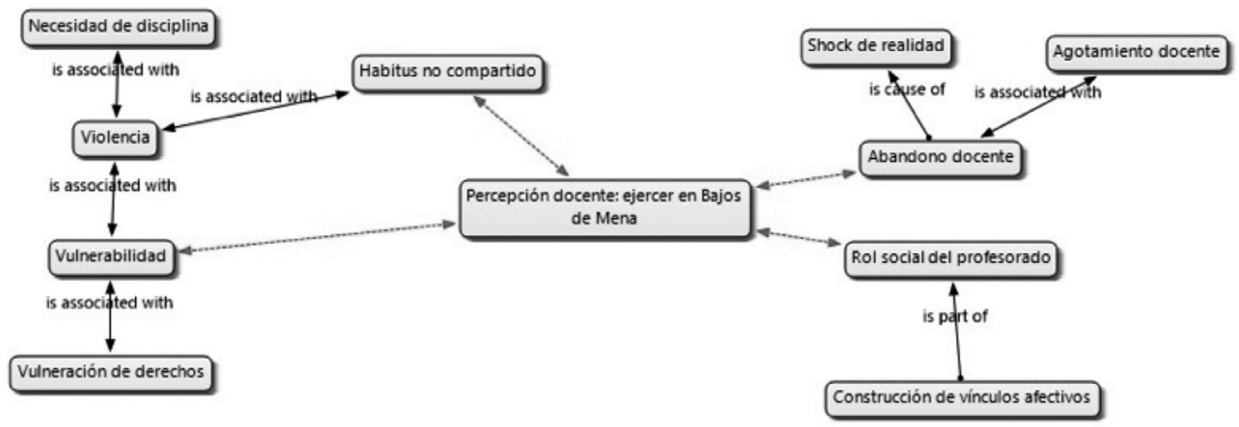

A partir de lo anterior, ejercer la docencia en Bajos de Mena en el imaginario de este profesor se vincula directamente con la violencia de los estudiantes y con el shock de realidad ${ }^{6}$ con el que se enfrenta el profesorado.

La violencia emerge de manera explícita e implícita al describir los códigos con los que el estudiantado se desenvuelve y relaciona al interior de la escuela, las pautas de interacción entre estudiantes y en la vinculación construida entre estudiantes y profesores. Por lo tanto, ejercer la docencia en estos lugares implica, como condición inherente, el manejo de la violencia.

Desde que se fundó [la escuela] nunca ha seleccionado. Siempre hemos recibido a todos los niños, y eso hace que sea un trabajo superdifícil [...] y hay niños que se escapan de los protocolos regulares, se ponen violentos o que los insultan.

Esta condición es lo que, a juicio del docente, pone en tensión a la profesión. En su experiencia, los profesores noveles no cuentan con las competencias suficientes para comprender la vulnerabilidad de los estudiantes y los códigos en los que se desenvuelven, interactúan y demandan la atención del profesorado.

Creo que cuando los chicos van a hacer práctica, hay un choque cultural superimportante porque donde trabajo el nivel de violencia verbal y física es superalta. Entonces

6 El shock de realidad se refiere al impacto que supone para los profesores enfrentarse a la realidad del aula y el mundo escolar, que difiere de ser un escenario ideal (INGERSOLl y STRONG, 2OII). 
los chicos que llegan a hacer sus prácticas se ven, primero, superimpactados. Y hay una falta de resguardo emocional para la persona que entra a trabajar a un sector así, de esas características. Entonces se ven, primero impactados por la realidad de los niños. Y segundo, cuando ellos tienen que implementar clase, y hay niños que se escapan de los protocolos digamos, regulares de, no sé, que se ponen violentos o que los insultan.

Sin embargo, la alusión al choque cultural parece especialmente relevante, y es lo que vincula a la categoría de habitus no compartido.

Si el habitus se refiere a ciertas prácticas, discursos e imaginarios que un grupo social comparte, el choque cultural entre profesores y estudiantes precisamente da cuenta del distanciamiento entre prácticas e imaginarios, generando una ruptura en la construcción social, ya que esta es no compartida (Duarte, 2012). Por lo tanto, independientemente de dónde provengan los profesores aludidos en el relato ${ }^{7}$, proponen formas o intentan educar desde una construcción social no compartida y, por ende, lejana de los estudiantes.

De hecho, el profesor hace alusión a protocolos regulares, los que no son definidos en el contexto de la entrevista, ya que se dan por entendidos. En este caso, entrevistado y entrevistador comparten el código de conducta que se valora como deseable en un determinado espacio escolar, sin embargo, este código no es el que predomina en la interacción natural del estudiantado. Por lo tanto, la violencia como condición natural para niños y jóvenes del entorno se aplaca a través de normas de disciplina que poco se relacionan con los modos culturales de estos estudiantes y el territorio.

Por lo tanto, la escuela como unidad en un determinado territorio actúa como ente socializador de una cultura que no representa a los estudiantes, y lo hace desde un código cultural distanciado (Berstein, I993). A esto, se suma la tensión que presenta la necesidad de generar instancias de clases al interior del aula que respondan a exigencias externas y nacionales, ya que a la estigmatización propia del lugar se le añade el karma de los malos resultados en el simce.

Por otra parte, el relato del docente avanza hacia una comprensión a partir de las carencias y la vulnerabilidad de los estudiantes, estableciendo una relación entre vulnerabilidad y vulneración de derechos. De esta forma, educar en Bajos de Mena, particularmente en esta escuela, supone para el profesorado moverse en una frágil zona de códigos encontrados y expuestos a situaciones que le pueden llevar a reponer derechos vulnerados por las condiciones sociales y territoriales que dibujan el entorno de la escuela.

\section{Algunas reflexiones sobre la segregación}

Educar en contextos de exclusión social implica adentrarse en un territorio desconocido, que no comparte el habitus normado y reproducido a través del sistema educacional formal. Sin embargo, para el profesorado ahí inserto las condiciones y exigencias le obligan a buscar formas que le permitan cumplir con las responsabilidades y estándares nacionales independientemente de lo complejo del contexto.

En el caso analizado, esta situación suma otra variable de complejidad. Al igual que con las políticas habitacionales, el territorio ha sido entregado a los intereses de

En Chile, en general, los profesores tienden a emplearse en escuelas de un nivel socioeconómico similar al de ellos, contribuyendo a reproducir círculos sociales (De los Ríos y VALenzuela, 2013). 
particulares que han creado proyectos educativos en esos espacios. Si bien estos espacios formativos son en su totalidad gratuitos para las familias, operan a través del sistema de subvenciones implementado en el sistema educacional chileno, dejando replegada a una mínima cuota la actuación directa del Estado.

Aunque estos espacios sean gratuitos no significa que: I) sean públicos, ya que los proyectos educativos representan intereses privados y la relación comunidad escuela es a través de un sostenedor, que es propietario y administrador del establecimiento; 2) presenten un proyecto educativo para esa realidad particular, ya que la vara de medición sigue siendo una prueba única nacional estandarizada para todos los estudiantes del territorio nacional, independientemente de su ubicación geográfica, condición económica o cultural; 3) propicien mecanismos orientados a contrarrestar la condición de marginalidad, estigmatización y criminalidad, ya que son escuelas a las que solo acceden niños del sector; 4) actúen como espacios promotores de equidad social, sino que en la práctica operan principalmente como sitios de contención de la violencia y prevención del delito.

El tiempo transcurrido ha favorecido la consolidación de una situación que a estas alturas parece irrevocable. Sin embargo, los miles de personas localizadas en los sectores marginales construidos exclusivamente para albergarlos y desplazarlos de los centros económicos y de poder necesitan cambios estructurales urgentes que les permitan acceder a los servicios, particularmente el servicio educativo. Este cambio es fundamental para la reposición de los derechos, mermados a partir de la imposición neoliberal sin coto, y para avanzar hacia una educación para la justicia social y realmente transformadora (Zeichner, 20I0; Apple, 20I8). De no avanzar en esa línea seguiremos mal educando desde habitus lejanos a los territorios en los que las escuelas se emplazan; seguiremos reproduciendo pobreza y conteniendo criminalidad, para que los estudiantes se transformen lo más tarde posible en los delincuentes que están condenados a ser (Ortega, 20I4).

Por ende, urge un Estado que deshaga su camino de abandono, desarrolle ciudad y forme realmente ciudadanos en equidad de derechos. Sin ello, Chile seguirá mostrando las cifras de desigualdad que hoy ostenta. Sin esto, todo esfuerzo del profesorado (aunque sea titánico) será marginal y de un impacto muy reducido.

\section{Bibliografía}

Apple, M. (20I8). ¿ Puede la educación cambiar la sociedad? (Traductora M. Valderrama). Santiago de Chile: Lom Ediciones.

Atria, F. (20io). ¿Qué «educación» es pública? En C. Bellei, D. Contreras y J. Valenzuela (eds.) Ecos de la revolución pingüina. Avances, debates y silencios en la reforma educacional. unicef, Universidad de Chile. Santiago de Chile: Pehuen Editores.

Bellei, C.; González, P. y Valenzuela, J. (20I0). Fortalecer la educación pública: un desafío de interés nacional. En C. Bellei, D. Contreras y J. Valenzuela (eds.) Ecos de la revolución pingüina. Avances, debates y silencios en la reforma educacional. UNICEF, Universidad de Chile. Santiago de Chile: Pehuen Editores.

Bernstein, B. (200I). La estructura del discurso pedagógico. Madrid, España: Ediciones Morata.

Bourdieu, P. (1997). Capital cultural, escuela y espacio social. Ciudad de México: Siglo xxi Editores.

Bravo, J. (20I4). Fondo Común Municipal y su desincentivo a la recaudación en Chile. Temas de la Agenda Pública, 9 (68), 5-18. 
Contreras, D. (1999). Distribución del ingreso en Chile. Nueve hechos y algunos mitos. Revista Perspectivas, 3II-332.

Cornejo, R.; Albornoz, N.; Castañeda, L.; Palacios, D.; Etcheberrigaray, G.; FernánDez, R.; Gómez, S.; Hidalgo, F. y Lagos, J. (2015). Las prescripciones del trabajo docente en el nuevo marco regulatorio de políticas educativas en Chile. Psicoperspectivas, I4(2), 7283. Recuperado de http://www.psicoperspectivas.cl https://doi.org/IO.5027/psicoperspectivas-voli4-issue2-fulltext-580.

Duarte, C. (20I2). Sociedades adultocéntricas: sobre sus orígenes y reproducción. Ultima Década, n. ${ }^{\circ}$ 36, cidpa Valparaíso, 99-I25.

Educación 2020 (20I7). ¿Cuánto ha avanzado la educación chilena en la última década? Y cuánto nos falta para llegar a la «meta». Recuperado de http://www.educacion2020.cl/sites/ default/files/estudio_ed._chilena_2008-2018.pdf.

Gallego, F. y SAPELli, C. (2007). El financiamiento de la educación en Chile: una evaluación. Rev. Pensamiento Educativo, 40(I), 263-284.

Garcés, M. (2012). El despertar de la sociedad. Los movimientos sociales en América Latina y Chile. Santiago de Chile: Lom.

GibBs, G. (2012). El análisis de datos cualitativos en Investigación Cualitativa. Madrid, España: Morata.

Grez, S. (1995). La cuestión social en Chile. Ideas y debates precursores (I804-1902). Santiago de Chile: Dirección de Bibliotecas, Archivos y Museos.

Hidalgo, R. (2007). ¿Se acabó el suelo en la gran ciudad? Las nuevas periferias metropolitanas de la vivienda social en Santiago de Chile. Revista Eure, 33(98), 57-75.

Hidalgo, R.; Urbina, P.; Alvarado, V. y Paulsen, A. (20I7). Desplazados y ¿olvidados?: contradicciones respecto de la satisfacción residencial en Bajos de Mena, Puente Alto, Santiago de Chile. Revista INVI, $32(89)$, 85-IIo.

Ingersoll, R. y Strong, M. (20II). The Impact of Induction and Mentoring Programs for Beginning Teachers: A Critical Review of. Review of Educational Research, 2(8I), 20I-233.

Martínez, A. (20I7). Invisibilización, procesos de guetización y negación del Derecho a la Ciudad: Población Bajos de Mena en la Comuna de Puente Alto, Santiago de Chile. BEGEO, (5), 55-68.

mineduc, Centro de Estudios. (2018). Indicadores de la Educación en Chile. 2010-20I6.

Ministerio de Educación Pública. (1973). Informe sobre Escuela Nacional Unificada. Santiago.

Morales, M. y López, V. Criminalización de la juventud en Chile: Políticas de convivencia escolar y traducciones locales. En G. Tenenbaum y N. Viscardi (coords.) Juventudes y violencias en América Latina. Sobre los dispositivos de coacción en el siglo XXI (pp. 173-I84). Montevideo, Uruguay: Ediciones Universitarias, Universidad de la República.

Ortega, T. (20I4). Criminalización y concentración de la pobreza urbana en barrios segregados. Síntomas de guetización en La Pintana, Santiago de Chile. Revista Eure, I20(40), $24 \mathrm{I}-263$.

PARdo, M. (20I3). La visión de los docentes sobre las reformas educacionales. En B. Ávalos ¿Héroes o villanos? La profesión docente en Chile (pp. 155-173). Santiago de Chile: Universitaria.

Piñuel, J. L. (2002). Epistemología, metodología y técnicas de análsis de contenido. Estudios de Sociolingüistica, 3(I), I-42.

PNUD. (20I7). Desiguales. Orígenes, cambios y desafíos de la brecha social en Chile. Santiago de Chile: Uqbar editores.

Puchi, N. (2017). Complejo Artístico Deportivo Bajos de Mena. Memoria para optar al título de Arquitecto. Universidad de Chile, Santiago.

Ríos, D. de y Valenzuela, J. (2013). Los docentes chlenos. Caracterización general sociodemográfica, cultural y laboral. En B. Ávalos ¿Héroes o villanos? La profesión docente en Chile (pp. 63-90). Santiago: Universitaria. 
Sabatini, F. y Wormald, G. (2013). Segregación de la vivienda social: reducción de oportunidades, pérdida de cohesión. En F. Sabatini, G. Wormald y A. Rasse (eds.) Segregación de la vivienda social: ocho conjuntos en Santiago, Concepción y Talca (pp. I2-3I). Santiago, Chile: Colección Estudios Urbanos uc.

Strauss, A. y Corbin, J. (2002). Bases de la investigación cualitativa. Técnicas y procedimientos para desarrollar teoría fundamentada. Antioquia: Universidad de Antioquia.

Tapia, R. (20II). Vivienda social en Santiago de Chile. Análisis de su comportamiento locacional, periodo I980 - 2002. Revista INVI, 73(26), I05-I3I.

Weinstein, J.; Fuenzalida, A. y Muñoz, G. (20I0). La subvención preferencial: desde una difícil instalación hacia su institucionalización. En S. MARTInIC y G. Elacqua (eds.) ¿̨in de ciclo? Cambios en la gobernanza del sistema educativo. Santiago: unesco, Universidad Católica.

Zeichner, K. (20Io). La formación del profesorado y la lucha por la justicia social. Madrid, España: Morata. 
